\title{
Differences in perceived parental stress between parents with very low birth weight infants and nurses in neonatal intensive care units, South Korea
}

\author{
Seol-Hee Moon ${ }^{1}$, Ho-Ran Park², Dong Yeon Kim ${ }^{3}$ \\ ${ }^{1}$ Registered Nurse, Neonatal Intensive Care Unit, Seoul St. Mary's Hospital, The Catholic University of Korea, Seoul; ${ }^{2}$ Professor Emeritus, College of Nursing, The \\ Catholic University of Korea, Seoul; ${ }^{3}$ Unit Manager, Hematology Intensive Care Unit, Seoul St. Mary's Hospital, The Catholic University of Korea, Seoul, Korea
}

Purpose: This descriptive study compared the perceived parental stress levels between parents with very low birth weight infants (VLBWIs) and nurses in the neonatal intensive care unit (NICU). Methods: In total, 83 parents of VLBWIs and 78 NICU nurses were enrolled. Data were collected with the Parental Stress Scale (PSS) and analyzed using the t-test and analysis of variance in SAS version 9.4. Results: The average PSS score was 3.31 among parents and 3.45 among nurses. The stress score was significantly higher among nurses with children $(\mathrm{t}=2.46, p=.016)$ and senior nurses $(\mathrm{t}=2.12, p=.037)$. There was a significant difference in the stress score according to parents' education ( $t=3.29, p=.002)$ and occupation $(\mathrm{F}=3.14, p=.049)$ in the sights and sounds subscale. Mothers had significantly higher stress scores than fathers in the parental role alterations subscale $(t=2.32$, $p=.023)$. Parental stress scores were higher than those perceived by nurses in the infant's appearance and behaviors subscale for breathing patterns ( $\mathrm{t}=2.95, p=.004)$, followed by jerky/ restless behavior ( $\mathrm{t}=2.70, p=.008)$. Conclusion: Nurses should provide explanations to parents of VLBWIs in order to reduce parental stress about the appearances and behavior of VLBWIs. This is more important than aspect of the NICU environment and education about parental roles.

Key words: Neonatal intensive care unit; Very low birth weight infants; Stress, psychological; Parents; Nurses

\section{Corresponding author \\ Dong Yeon Kim}

Hematology Intensive Care Unit, Seoul

St. Mary's Hospital, The Catholic

University of Korea, 222 Banpo-daero,

Seocho-gu, Seoul 06591, Korea

TEL: +82-2-2258-5353

FAX: $+82-2-2258-6544$

E-MAIL: vonma98@naver.com

This article was adapted from a thesis by Seol-Hee Moon in partial fulfillment of the requirements for the master's degree at the Catholic University of Korea.

Received Jun 12, 2021

Revised Jun 26, 2021

Accepted Jul 14, 2021

\begin{abstract}
This is an Open Access article distributed under the terms of the Creative Commons Attribution NonCommercial of the Creative Commons Attribution NonCommercial
License (http://creativecommons.org/licenses/by-nc/4.0/) which permits unrestricted noncommercial use, distribution, and reproduction in any medium, provided the original work is properly cited.
\end{abstract}

\section{INTRODUCTION}

\section{Need for Study}

With advances in medical technology, older age at marriage and childbirth, and an increased rate of infertility procedures, the number of very low birth weight infants (VLBWIs) born before gestational week 37 is dramatically rising over time [1-4]. As VLBWIs have immature body organs, they begin their life's journey in a neonatal intensive care unit (NICU) for an indeterminate period of time $[5,6]$. The birth of a child is a stressor for parents [7], and the infant's admission to the NICU following birth is a greater stressor $[3,4,8]$. Mothers with babies in the NICU experience many stressors due to prematurity, the infants' medical or surgical condition, the stressful NICU environment, and anticipated vulnerability apart from the psychological morbidity perceived during pregnancy and the perinatal transition [9]. Bright lights, noisy life support and monitoring devices, tubes, wire connections, intravenous catheters, and the continuous presence of nurses are thought to be the main elements that disturb parents. Visits (including those made by parents) are restricted for high-risk neonates who require highly advanced treatment and care during the NICU stay per hospital policy, so parents cannot hold their newborns; instead, nurses provide care for newborns, hindering normal family time [5].

As parents go through the disease and treatment process involving the hospitalization of their newborn, they become increasingly anxious about the unpredicted state of their infant and the uncertain future, which in turn induces psychological and mental stress and consequent confusion and helplessness, resulting in a loss of confidence in parenting $[6,10]$. 
Nurses working in the NICU need to recognize the importance of infant safety felt by newborn mothers, and it is necessary to show the mother a comfortable appearance of her infant during visits whenever possible [10]. Parental stress is very often neglected, as attention is focused mainly on the care and survival of sick neonates [9]. As discussed in Yu's study [11], considering that most NICU nurses are young and have never given birth, it may be difficult for them to adequately empathize with parents who would be torn as they witness their infant being taken to the NICU and striving to overcome their physical challenges and illnesses.

Parents of NICU-admitted babies are under significant stress, which needs to be identified early along with care for the sick neonate, in order to provide holistic care to the family. Nursing support is essential for mothers of premature infants, as a way for parents to obtain information about the infant's treatment process, receive comfort, and regain their self-esteem as parents [12].

Previous domestic studies on nursing care and the experiences of parents of high-risk newborns include a study on the comparison of parental stress [13], a study on fathers' stress and nursing support [5], and a study on relationship between nurse's communication and parental stress [4]. To date, the majority of studies [14-16] have focused on understanding sources of stress using the Parental Stressor Scale: Neonatal Intensive Care Unit (PSS:NICU), which is designed to measure parental perceptions of stressors arising from the physiological and psychosocial environment of the NICU. In previous research, it was found that scores on the infant's appearance and behaviors subscale of the PSS:NICU reflected the highest rate of stress. It is therefore necessary to investigate whether nurses also experience a high level of stress in the same domains as parents of VLBWIs.

Stress responses among parents of premature infants experiencing the NICU environment have been widely reported. However, less is known about how nurses perceive parents' experiences associated with VLBWIs hospitalization and parental stress levels in the NICU. The difference between the stress perceived by parents and nurses will indicate what kind of education and nursing support parents need to receive.

Thus, this study aimed to examine the level of stress among parents of VLBWIs and the gap between parents' and nurses' stress scores to present foundational data for developing nursing intervention programs to alleviate parents' stress.

\section{Purpose}

This study had the following objectives: to quantify the stress of parents of VLBWIs; to quantify perceived parental stress among nurses who provide care for VLBWIs; to identify differences in stress according to the general characteristics of parents of VLBWIs; to identify differences in stress according to the general characteristics of nurses who provide care for VLBWIs; and to compare the differences between stress experienced by parents of VLBWIs and that perceived by nurses.

\section{METHODS}

Ethics statement: This study was approved by the Institutional Review Board of Catholic University of Korea (No. XC16QAMI0082K). Informed consent was obtained from the participants.

\section{Study Design}

This descriptive survey aimed to examine and compare the levels of stress experienced by parents of VLBWIs and parental stress perceived by NICU nurses.

\section{Subjects}

Parents of VLBWIs admitted to the NICU in three university- affiliated hospitals and nurses with at least 1 year of career experience in the NICU were enrolled in the study. The sample size was determined using $\mathrm{G}^{*}$ Power 3.1.9.2 [17]. The minimum sample size was 146 when calculated based on the t-test at a significance of .05, effect size of .5, and power of .85 . Anticipating a $10 \%-20 \%$ potential drop- out rate, 184 participants were recruited. The responses of nine participants from the parent group and 10 participants from the nurse group were not analyzed because they were incomplete, and four nurses discontinued participation in the study. Therefore, a total of 161 participants (83 parents and 78 nurses) were included in the analysis.

\section{Study Tool}

\section{1) Parental Stressor Scale: Neonatal Intensive Care Unit}

Stress of parents of VLBWIs was measured using the PSS: NICU, which was developed by Miles et al. [18] to measure stress among parents of newborns admitted to the NICU. In this study, the translated version by Kim [19] was partially modified and adapted for use in the NICU with permission from the original author. The phrases were changed "my infant" to "infant", and "I" to "parents" to allow parents and nurses to use the same tool. The content validity index (CVI) was calculated for all individual items (I-CVI) for 45 items. To determine the CVI, a panel of experts was asked to rate each scale item in terms of its relevance to the underlying construct. The panel consisted of one nursing professor and seven clin- 
ical nursing professionals with a master's degree with at least 5 years of NICU career experience at a tertiary hospital. A 4-point scale was used to avoid a neutral point, and the I-CVI was .86 .

The PSS:NICU is a 45 -item instrument that measures parents' perceptions of stress within the NICU. The scale consists of four subscales that measure stress related to sights and sounds (5 items), the infant's appearance and behaviors (19 items), parental role alterations (10 items), and communication with health care providers (11 items). It also has a question about general stress that summarizes 'parents' overall feeling of stress related to having an infant in the NICU. Each subscale is rated on a 6-point scale from "no stress at all" (0) to "extremely stressful" (5). The scores range from 0 to 5 points, with a higher score indicating greater stress.

Cronbach's $\alpha$ was .94 in the study by Miles et al. [18], .93 in the study by Kim [19], and .96 in this study. By subscales, Cronbach's $\alpha$ was .81 for sights and sounds .81, .95 for infant's appearance and behaviors, .93 for parental role alterations, and .95 for communication with health care providers. Cronbach's $\alpha$ was .96 for parental stress and .97 for nurses' awareness.

\section{Data Collection}

Data were collected from January 25, 2017 to September 30, 2017. After obtaining permission from the nursing departments at three hospitals, the principal investigator provided an explanation of the purpose of the study, distributed the questionnaires to parents and nurses who provided consent, and collected the completed questionnaires in an envelope. After obtaining informed consent from the parents and nurses, the questionnaire was completed anonymously. It took approximately 10-15 minutes to complete the questionnaire.

\section{Data Analysis}

The collected data were analyzed using SAS for Windows version 9.4 (SAS Institute Inc., Cary, NC, USA). Descriptive statistics were used to examine demographic characteristics and perceived parental stress among parents of VLBWIs and nurses. The demographic characteristics for parents and nurses showed a normal distribution. The level of perceived parental stress of parents of VLBWIs and nurses according to their general characteristics was analyzed using the independent $t$-test and one-way analysis of variance (ANOVA). Posthoc testing was performed with the Scheffé test for significant results in ANOVA. Perceived parental stress was compared between parents of VLBWIs and nurses using the independent t-test.

\section{Ethical Considerations}

The content and method of this study were approved by the Institutional Review Board (IRB) of the Catholic University of Korea (No. XC16QAMI0082K). The participants were informed about the protection of personal information, confidentiality, use of data only for research purposes, and their freedom to withdraw from the study at any time before asking them to sign the consent form. Only numbers were used on the questionnaire without any personal information to ensure anonymity. The participants were given IRB contact information to direct any inquiries not resolved by the researchers, concerns, complaints, or questions about their rights, and participants who completed the questionnaire were given a prepared gift.

\section{RESULTS}

\section{General Characteristics and Stress of Parents with VLBWIs}

Parents' mean stress score was 3.31. There were 50 mothers $(60.2 \%)$ and 33 fathers (39.8\%), and the mean age was 34.6 years, with $38(45.8 \%)$ participants aged 36 or older (range, 21-49 years). Most of the participants were university graduates or higher $(n=69,83.1 \%)$, and $36(43.4 \%)$ worked in business. The most common average monthly income was 6 million South Korean won or higher $(n=27,32.5 \%)$. Forty-seven $(56.6 \%)$ of the infants were female. The most common type of pregnancy was natural pregnancy $(n=60,72.3 \%)$, while $23(27.7 \%)$ used artificial insemination. The mean gestation period was 27.8 weeks, and the mean birth weight was $997.7 \mathrm{~g}$. Parents with an infant with a birth weight of less than $600 \mathrm{~g}$ showed the highest stress scores (3.56), and parents who are high school graduates or lower showed the lowest stress scores (3.09). However, this difference was not statistically significant (Table 1).

\section{Nurses' General Characteristics and Perceived Parental Stress}

All nurses who provided care for VLBWIs were women, and the mean perceived parental stress was 3.45 points. Nurses' mean age was 28.7 years, with the greatest number of nurses between the ages of 26 and 29 years $(n=29,37.2 \%)$ (range, 23-55 years). Fifty-four nurses (69.2\%) were single, and 61 $(78.2 \%)$ did not have a child.

The mean total clinical career experience was 6.2 years, and most had less than 5 years of career experience $(n=43,55.2 \%)$. The mean NICU career experience was 5.4 years, with most having less than 5 years of NICU experience $(n=45,57.7 \%)$. The majority of the nurses were staff nurses $(n=63,80.8 \%)$, 
Table 1. General Characteristics and Stress of Parents with VLBWIs $(N=83)$

\begin{tabular}{|c|c|c|c|c|c|c|}
\hline \multirow{2}{*}{ Characteristics } & \multirow{2}{*}{ Categories } & \multirow{2}{*}{$\mathrm{n}(\%)$} & \multirow{2}{*}{$\mathrm{M} \pm \mathrm{SD}$} & Stress level & \multirow{2}{*}{ t or $F$} & \multirow{2}{*}{$p$} \\
\hline & & & & $\mathrm{M} \pm \mathrm{SD}$ & & \\
\hline Total stress & & & & $3.31 \pm 0.72$ & & \\
\hline Parents & $\begin{array}{l}\text { Father } \\
\text { Mother }\end{array}$ & $\begin{array}{l}33(39.8) \\
50(60.2)\end{array}$ & & $\begin{array}{l}3.19 \pm 0.71 \\
3.39 \pm 0.73\end{array}$ & 1.27 & .207 \\
\hline Age (year) & $\begin{array}{l}<30 \\
\geq 30 \&<36 \\
\geq 36\end{array}$ & $\begin{array}{l}14(16.8) \\
31(37.4) \\
38(45.8)\end{array}$ & $34.6 \pm 5.3$ & $\begin{array}{l}3.17 \pm 0.88 \\
3.34 \pm 0.68 \\
3.34 \pm 0.72\end{array}$ & 0.31 & .736 \\
\hline Education level & $\begin{array}{l}\leq \text { High school graduate } \\
\geq \text { University graduate }\end{array}$ & $\begin{array}{l}14(16.9) \\
69(83.1)\end{array}$ & & $\begin{array}{l}3.09 \pm 0.83 \\
3.36 \pm 0.70\end{array}$ & 1.24 & .220 \\
\hline Occupation & $\begin{array}{l}\text { Office work } \\
\text { Profession } \\
\text { Business }\end{array}$ & $\begin{array}{l}29(34.9) \\
18(21.7) \\
36(43.4)\end{array}$ & & $\begin{array}{l}3.31 \pm 0.63 \\
3.44 \pm 0.70 \\
3.24 \pm 0.82\end{array}$ & 0.42 & .660 \\
\hline $\begin{array}{l}\text { Monthly income } \\
(10,000 \text { won })\end{array}$ & $\begin{array}{l}<240 \\
\geq 240 \&<350 \\
\geq 350 \&<500 \\
\geq 500 \&<600 \\
\geq 600\end{array}$ & $\begin{array}{c}8(9.6) \\
16(19.3) \\
17(20.5) \\
15(18.1) \\
27(32.5)\end{array}$ & & $\begin{array}{l}3.21 \pm 0.60 \\
3.26 \pm 1.03 \\
3.40 \pm 0.62 \\
3.34 \pm 0.64 \\
3.30 \pm 0.69\end{array}$ & 0.13 & .970 \\
\hline Infant's sex & $\begin{array}{l}\text { Male } \\
\text { Female }\end{array}$ & $\begin{array}{l}36(43.4) \\
47(56.6)\end{array}$ & & $\begin{array}{l}3.28 \pm 0.69 \\
3.34 \pm 0.76\end{array}$ & 0.37 & .715 \\
\hline Pregnancy & $\begin{array}{l}\text { Natural } \\
\text { Artificial }\end{array}$ & $\begin{array}{l}60(72.3) \\
23(27.7)\end{array}$ & & $\begin{array}{l}3.27 \pm 0.72 \\
3.43 \pm 0.73\end{array}$ & 0.92 & .361 \\
\hline $\begin{array}{l}\text { Gestational age of infant } \\
\text { (week) }\end{array}$ & $\begin{array}{l}<25 \\
\geq 25 \&<30 \\
\geq 30\end{array}$ & $\begin{array}{l}15(18.1) \\
46(55.4) \\
22(26.5)\end{array}$ & $27.8 \pm 3.1$ & $\begin{array}{l}3.42 \pm 0.73 \\
3.34 \pm 0.77 \\
3.18 \pm 0.63\end{array}$ & 0.53 & .590 \\
\hline Infant's birth weight (g) & $\begin{array}{l}<600 \\
\geq 600 \&<1,000 \\
\geq 1,000\end{array}$ & $\begin{array}{c}8(9.6) \\
37(44.6) \\
38(45.8)\end{array}$ & $997.7 \pm 312.2$ & $\begin{array}{l}3.56 \pm 0.47 \\
3.30 \pm 0.73 \\
3.27 \pm 0.77\end{array}$ & 0.53 & .590 \\
\hline
\end{tabular}

VLBWIs, very low birth weight infants.

and $71(91.0 \%)$ were Bachelor's degree graduates or lower.

The mean perceived parental stress score was 3.82 among nurses with a child, which was significantly higher than that among nurses without a child (3.35) $(\mathrm{t}=2.46, p=.016)$. Furthermore, the mean perceived parental stress score was significantly higher among charge nurses or higher (3.80) than among staff nurses (3.37) $(\mathrm{t}=2.12, p=.037)$ (Table 2).

\section{Comparison of Stress by Subscale According to the General Characteristics of Parents with VLBWls}

While there were no significant differences in the total stress score according to the characteristics of parents of VLBWIs, there were significant differences by subscale (Table 3 ).

Scores in the sights and sounds subscale showed significant differences according to parents' education level and occupation. The mean score was significantly higher among university graduates or higher (2.89) than among high school gradu- ates (2.06) $(\mathrm{t}=3.29, p=.002)$ and among professionals (3.17) than office workers (2.77) and those who worked in business (2.51) $(\mathrm{F}=3.14, p=.049)$. The average stress score in the parental role alterations subscale was significantly higher among mothers (3.38) than among fathers (2.90) $(\mathrm{t}=2.32, p=.023)$.

\section{Perceived Parental Stress by Subscale According to Nurses' Characteristics}

The differences in nurses' perceived parental stress according to their general characteristics are shown in Table 4 . The stress score in the sights and sounds subscale significantly differed according to marital status, child status, total clinical career, NICU career, and working position. The perceived parental stress score was higher among married nurses (3.73) than among single nurses (3.31) $(\mathrm{t}=2.66, p=.010)$ and among nurses with a child (3.92) than among those without a child (3.30) $(\mathrm{t}=3.66, p<.001)$. The perceived parental stress score was 
Table 2. Nurses' General Characteristics and Perceived Stress of Parents of VLBWIs $(N=78)$

\begin{tabular}{|c|c|c|c|c|c|c|}
\hline \multirow{2}{*}{ Characteristics } & \multirow{2}{*}{ Categories } & \multirow{2}{*}{$\mathrm{n}(\%)$} & \multirow{2}{*}{$\mathrm{M} \pm \mathrm{SD}$} & Stress level & \multirow{2}{*}{ tor $F$} & \multirow{2}{*}{$p$} \\
\hline & & & & $\mathrm{M} \pm \mathrm{SD}$ & & \\
\hline Total stress & & & & $3.45 \pm 0.72$ & & \\
\hline Gender & $\begin{array}{l}\text { Male } \\
\text { Female }\end{array}$ & $\begin{array}{c}0(0.0) \\
78(100.0)\end{array}$ & & $\begin{array}{c}- \\
3.45 \pm 0.72\end{array}$ & & \\
\hline Age (year) & $\begin{array}{l}<26 \\
\geq 26 \&<30 \\
\geq 30 \&<36 \\
\geq 36\end{array}$ & $\begin{array}{r}25(32.1) \\
29(37.2) \\
15(19.2) \\
9(11.5)\end{array}$ & $28.7 \pm 5.6$ & $\begin{array}{l}3.32 \pm 0.74 \\
3.42 \pm 0.72 \\
3.60 \pm 0.49 \\
3.71 \pm 0.93\end{array}$ & 0.88 & .455 \\
\hline Marital status & $\begin{array}{l}\text { Single } \\
\text { Married }\end{array}$ & $\begin{array}{l}54(69.2) \\
24(30.8)\end{array}$ & & $\begin{array}{l}3.40 \pm 0.74 \\
3.58 \pm 0.66\end{array}$ & 1.06 & .294 \\
\hline With children & $\begin{array}{l}\text { No } \\
\text { Yes }\end{array}$ & $\begin{array}{l}61(78.2) \\
17(21.8)\end{array}$ & & $\begin{array}{l}3.35 \pm 0.73 \\
3.82 \pm 0.56\end{array}$ & 2.46 & .016 \\
\hline Career (year) & $\begin{array}{l}<5 \\
\geq 5 \&<10 \\
\geq 10\end{array}$ & $\begin{array}{l}43(55.2) \\
20(25.6) \\
15(19.2)\end{array}$ & $6.2 \pm 5.3$ & $\begin{array}{l}3.36 \pm 0.79 \\
3.40 \pm 0.58 \\
3.80 \pm 0.61\end{array}$ & 2.24 & .113 \\
\hline NICU career (year) & $\begin{array}{l}<5 \\
\geq 5 \&<10 \\
\geq 10\end{array}$ & $\begin{array}{l}45(57.7) \\
19(24.3) \\
14(18.0)\end{array}$ & $5.4 \pm 4.8$ & $\begin{array}{l}3.36 \pm 0.77 \\
3.39 \pm 0.59 \\
3.83 \pm 0.62\end{array}$ & 2.41 & .097 \\
\hline Working position & $\begin{array}{l}\text { Staff nurse } \\
\geq \text { Charge nurse }\end{array}$ & $\begin{array}{l}63(80.8) \\
15(19.2)\end{array}$ & & $\begin{array}{l}3.37 \pm 0.72 \\
3.80 \pm 0.61\end{array}$ & 2.12 & .037 \\
\hline Education level & $\begin{array}{l}\leq \text { Bachelor's degree graduate } \\
\geq \text { Master's degree graduate }\end{array}$ & $\begin{array}{l}71(91.0) \\
7(9.0)\end{array}$ & & $\begin{array}{l}3.42 \pm 0.71 \\
3.80 \pm 0.74\end{array}$ & 1.35 & .179 \\
\hline
\end{tabular}

NICU, neonatal intensive care unit; VLBWIs, very low birth weight infants.

also higher among nurses with a clinical career or 10 years or longer (3.81) than among those with less than 5 years (3.29) or 5 to 9 years of experience (3.48) $(\mathrm{F}=3.86, p=.026)$. The perceived parental stress score was higher among nurses with a NICU career or 10 years or longer (3.86) than among those with less than 5 years (3.27) or 5 to 9 years of experience (3.53) $(\mathrm{F}=4.91, p=.010)$.

The perceived parental stress score was also higher among charge nurses or higher (3.81) than among staff nurses (3.35) $(\mathrm{t}=2.53, p=.013)$. The perceived parental stress score in the infant's appearance and behaviors subscale significantly differed according to nurses' characteristics, and those with a child perceived that parents were more stressed (3.97) than those without a child (3.50) $(\mathrm{t}=2.19, p=.032)$. In the communication with health care providers subscale, charge nurses or higher perceived significantly greater parental stress (3.78) than staff nurses (3.25) $(\mathrm{t}=2.42, p=.018)$.

\section{Comparison of Stress of Parents with VLBWls and NICU Nurses' Perceived Parental Stress}

There were no differences between the overall stress of parents of VLBWIs (3.31 out of 5) and nurses' perceived parental stress (3.45 out of 5) (Table 2). However, there were significant differences in the stress scores for some subscales (Table 5).

\section{1) Sights and sounds}

In the sights and sounds subscale, significant differences were found in the stress score between parents (2.75) and nurses (3.44) $(\mathrm{t}=5.48, p<.001)$. Parents showed the greatest stress for the "sudden noise /alarm" item (3.60) and nurses also perceived parents would have the greatest stress in that situation (4.19). However, nurses perceived that parents would be more stressed than they actually were $(t=3.65, p<.001)$ (Table 5).

\section{2) Infant's appearance and behaviors}

In the infant's appearance and behaviors subscale, there were no significant differences in the stress score between parents (3.78) and nurses (3.60). However, unlike other subscales, parents' stress score was higher than nurses' perceived parental stress score. Parents showed greater stress than nurses' perceived stress scores for the following items: "pale/jaundiced" $(\mathrm{t}=$ 3.68, $p<.001)$, "breathing pattern" $(\mathrm{t}=2.95, p=.004)$, "pain" $(\mathrm{t}=2.37$, $p=.019)$, "sad" $(\mathrm{t}=2.48, p=.014)$, "limp and weak" $(\mathrm{t}=3.06, p=$ $.003)$, "jerky / restless" $(\mathrm{t}=2.70, p=.008)$, and "can't cry" $(\mathrm{t}=1.99$, $p=.048)$. Parents showed less stress than nurses' perceived 
Table 3. Comparison of Stress by Subscale According to the General Characteristics of Parents with VLBWIs $(N=83)$

\begin{tabular}{|c|c|c|c|c|c|c|c|c|c|c|}
\hline \multirow[t]{2}{*}{ Characteristics } & \multirow[t]{2}{*}{ Categories } & \multirow[t]{2}{*}{ n (\%) } & \multicolumn{2}{|c|}{$\begin{array}{l}\text { Sights and } \\
\text { sounds }\end{array}$} & \multicolumn{2}{|c|}{$\begin{array}{c}\text { Infant's } \\
\text { appearance } \\
\text { and behaviors }\end{array}$} & \multicolumn{2}{|c|}{$\begin{array}{l}\text { Parental role } \\
\text { alterations }\end{array}$} & \multicolumn{2}{|c|}{$\begin{array}{l}\text { Communication } \\
\text { with health care } \\
\text { providers }\end{array}$} \\
\hline & & & $\mathrm{M} \pm \mathrm{SD}$ & t or $F(p)$ & $\mathrm{M} \pm \mathrm{SD}$ & $\mathrm{t}$ or $\mathrm{F}(p)$ & $\mathrm{M} \pm \mathrm{SD}$ & t or $F(p)$ & $\mathrm{M} \pm \mathrm{SD}$ & t or $F(p)$ \\
\hline Total stress & & $83(100.0)$ & $2.75 \pm 0.92$ & & $3.78 \pm 0.71$ & & $3.19 \pm 0.96$ & & $2.87 \pm 1.06$ & \\
\hline Parents & $\begin{array}{l}\text { Father } \\
\text { Mother }\end{array}$ & $\begin{array}{l}33(39.8) \\
50(60.2)\end{array}$ & $\begin{array}{l}2.70 \pm 0.93 \\
2.79 \pm 0.92\end{array}$ & $\begin{array}{l}0.44 \\
(.661)\end{array}$ & $\begin{array}{l}3.62 \pm 0.71 \\
3.88 \pm 0.70\end{array}$ & $\begin{array}{l}1.62 \\
(.019)\end{array}$ & $\begin{array}{l}2.90 \pm 0.98 \\
3.38 \pm 0.91\end{array}$ & $\begin{array}{c}2.32 \\
(.023)\end{array}$ & $\begin{array}{l}2.92 \pm 0.96 \\
2.84 \pm 1.12\end{array}$ & $\begin{array}{l}0.35 \\
(.729)\end{array}$ \\
\hline Age & $\begin{array}{l}<30 \\
\geq 30 \&<36 \\
\geq 36\end{array}$ & $\begin{array}{l}14(16.8) \\
31(37.4) \\
38(45.8)\end{array}$ & $\begin{array}{l}2.79 \pm 0.89 \\
2.61 \pm 0.77 \\
2.85 \pm 1.04\end{array}$ & $\begin{array}{l}0.59 \\
(.558)\end{array}$ & $\begin{array}{l}3.57 \pm 0.89 \\
3.89 \pm 0.63 \\
3.76 \pm 0.71\end{array}$ & $\begin{array}{l}1.00 \\
(.374)\end{array}$ & $\begin{array}{l}3.25 \pm 0.92 \\
3.18 \pm 1.02 \\
3.18 \pm 0.94\end{array}$ & $\begin{array}{l}0.03 \\
(.969)\end{array}$ & $\begin{array}{l}2.58 \pm 1.25 \\
2.87 \pm 1.05 \\
2.99 \pm 1.00\end{array}$ & $\begin{array}{l}0.73 \\
(.484)\end{array}$ \\
\hline $\begin{array}{l}\text { Education } \\
\text { level }\end{array}$ & $\begin{array}{l}\leq \text { High school graduate } \\
\geq \text { University graduate }\end{array}$ & $\begin{array}{l}14(16.9) \\
69(83.1)\end{array}$ & $\begin{array}{l}2.06 \pm 0.97 \\
2.89 \pm 0.85\end{array}$ & $\begin{array}{l}3.29 \\
(.002)\end{array}$ & $\begin{array}{l}3.48 \pm 0.89 \\
3.84 \pm 0.66\end{array}$ & $\begin{array}{l}1.72 \\
(.089)\end{array}$ & $\begin{array}{l}3.16 \pm 0.99 \\
3.20 \pm 0.96\end{array}$ & $\begin{array}{l}0.11 \\
(.912)\end{array}$ & $\begin{array}{l}2.83 \pm 1.05 \\
2.88 \pm 1.07\end{array}$ & $\begin{array}{l}0.16 \\
(.872)\end{array}$ \\
\hline Occu & $\begin{array}{l}\text { Office work } \\
\text { Profession }^{\mathrm{b}} \\
\text { Business }^{c}\end{array}$ & $\begin{array}{l}29(34.9) \\
18(21.7) \\
36(43.4)\end{array}$ & $\begin{array}{l}2.77 \pm 0.82 \\
3.17 \pm 0.77 \\
2.51 \pm 1.01\end{array}$ & $\begin{array}{c}3.14 \\
(.049) \\
b>a, c^{*}\end{array}$ & $\begin{array}{l}3.70 \pm 0.66 \\
4.12 \pm 0.53 \\
3.65 \pm 0.80\end{array}$ & $\begin{array}{l}2.81 \\
(.067)\end{array}$ & $\begin{array}{l}3.13 \pm 0.93 \\
3.18 \pm 1.05 \\
3.23 \pm 0.97\end{array}$ & $\begin{array}{l}0.10 \\
(.909)\end{array}$ & $\begin{array}{l}3.04 \pm 0.80 \\
2.63 \pm 1.21 \\
2.87 \pm 1.18\end{array}$ & $\begin{array}{c}0.81 \\
(.450)\end{array}$ \\
\hline $\begin{array}{l}\text { Monthly } \\
\text { income } \\
\text { (10,000 won) }\end{array}$ & $\begin{array}{l}<240 \\
\geq 240 \&<350 \\
\geq 350 \&<500 \\
\geq 500 \&<600 \\
\geq 600\end{array}$ & $\begin{array}{c}8(9.6) \\
16(19.3) \\
17(20.5) \\
15(18.1) \\
27(32.5)\end{array}$ & $\begin{array}{l}2.68 \pm 1.37 \\
2.83 \pm 1.12 \\
2.81 \pm 0.52 \\
2.80 \pm 0.78 \\
2.67 \pm 0.96\end{array}$ & $\begin{array}{l}0.12 \\
(.975)\end{array}$ & $\begin{array}{l}3.53 \pm 0.65 \\
3.67 \pm 0.98 \\
3.80 \pm 0.66 \\
3.86 \pm 0.47 \\
3.85 \pm 0.70\end{array}$ & $\begin{array}{l}0.44 \\
(.777)\end{array}$ & $\begin{array}{l}3.43 \pm 0.65 \\
3.31 \pm 1.03 \\
3.29 \pm 1.07 \\
3.16 \pm 0.95 \\
3.01 \pm 0.96\end{array}$ & $\begin{array}{l}0.46 \\
(.766)\end{array}$ & $\begin{array}{l}2.70 \pm 0.81 \\
2.71 \pm 1.56 \\
3.10 \pm 0.74 \\
2.86 \pm 1.14 \\
2.88 \pm 0.93\end{array}$ & $\begin{array}{l}0.33 \\
(.854)\end{array}$ \\
\hline Infant's & $\begin{array}{l}\text { Male } \\
\text { Female }\end{array}$ & $\begin{array}{l}36(43.4) \\
47(56.6)\end{array}$ & $\begin{array}{l}2.81 \pm 0.68 \\
2.71 \pm 1.07\end{array}$ & $\begin{array}{l}0.49 \\
(.624)\end{array}$ & $\begin{array}{l}3.80 \pm 0.60 \\
3.76 \pm 0.79\end{array}$ & $\begin{array}{l}0.29 \\
(.771)\end{array}$ & $\begin{array}{l}3.14 \pm 1.03 \\
3.23 \pm 0.92\end{array}$ & $\begin{array}{l}0.43 \\
(.671)\end{array}$ & $\begin{array}{l}2.71 \pm 1.13 \\
2.99 \pm 1.00\end{array}$ & $\begin{array}{l}0.12 \\
(.235)\end{array}$ \\
\hline Pregnancy & $\begin{array}{l}\text { Natural } \\
\text { Artificial }\end{array}$ & $\begin{array}{l}60(72.3) \\
23(27.7)\end{array}$ & $\begin{array}{l}2.81 \pm 0.98 \\
2.61 \pm 0.74\end{array}$ & $\begin{array}{l}0.88 \\
(.382)\end{array}$ & $\begin{array}{l}3.70 \pm 0.73 \\
3.97 \pm 0.64\end{array}$ & $\begin{array}{l}1.56 \\
(.124)\end{array}$ & $\begin{array}{l}3.22 \pm 0.85 \\
3.11 \pm 1.22\end{array}$ & $\begin{array}{l}0.41 \\
(.685)\end{array}$ & $\begin{array}{l}2.76 \pm 1.08 \\
3.16 \pm 0.97\end{array}$ & $\begin{array}{l}1.53 \\
(.129)\end{array}$ \\
\hline $\begin{array}{l}\text { Gestational age } \\
\text { of infant } \\
\text { (week) }\end{array}$ & $\begin{array}{l}<25 \\
\geq 25 \&<30 \\
\geq 30\end{array}$ & $\begin{array}{l}15(18.1) \\
46(55.4) \\
22(26.5)\end{array}$ & $\begin{array}{l}2.81 \pm 1.08 \\
2.81 \pm 0.88 \\
2.58 \pm 0.91\end{array}$ & $\begin{array}{l}0.51 \\
(.604)\end{array}$ & $\begin{array}{l}3.99 \pm 0.61 \\
3.77 \pm 0.76 \\
3.65 \pm 0.65\end{array}$ & $\begin{array}{l}1.00 \\
(.371)\end{array}$ & $\begin{array}{l}3.27 \pm 1.02 \\
3.28 \pm 0.93 \\
2.95 \pm 0.99\end{array}$ & $\begin{array}{c}0.97 \\
(.382)\end{array}$ & $\begin{array}{l}2.84 \pm 1.10 \\
2.89 \pm 1.15 \\
2.87 \pm 0.85\end{array}$ & $\begin{array}{c}0.01 \\
(.991)\end{array}$ \\
\hline $\begin{array}{l}\text { Infant's birth } \\
\text { weight (g) }\end{array}$ & $\begin{array}{l}<600 \\
\geq 600 \&<1,000 \\
\geq 1,000\end{array}$ & $\begin{array}{c}8(9.6) \\
37(44.6) \\
38(45.8)\end{array}$ & $\begin{array}{l}2.65 \pm 0.73 \\
2.72 \pm 0.96 \\
2.80 \pm 0.93\end{array}$ & $\begin{array}{l}0.12 \\
(.891)\end{array}$ & $\begin{array}{l}4.12 \pm 0.54 \\
3.79 \pm 0.68 \\
3.69 \pm 0.76\end{array}$ & $\begin{array}{l}1.22 \\
(.302)\end{array}$ & $\begin{array}{l}3.43 \pm 0.56 \\
3.17 \pm 1.02 \\
3.16 \pm 0.98\end{array}$ & $\begin{array}{c}0.26 \\
(.771)\end{array}$ & $\begin{array}{l}3.14 \pm 0.70 \\
2.82 \pm 0.99 \\
2.87 \pm 1.19\end{array}$ & $\begin{array}{l}0.29 \\
(.747)\end{array}$ \\
\hline
\end{tabular}

*Scheffé test, $p<.050$; NICU, neonatal intensive care unit; VLBWIs, very low birth weight infants.

stress score for the "tubes/equipment" item $(\mathrm{t}=2.04, p=.043)$ (Table 5).

\section{3) Parental role alterations}

In the parental role alterations subscale, there were no significant differences in the stress score between parents (3.19) and nurses (3.29). Parents showed lower stress (1.52) than nurses' perceived parental stress (2.68) when "medical staff closer to the infant than me" $(\mathrm{t}=5.86, p<.001)$ (Table 5).

\section{4) Communication with health care providers}

In the communication with health care providers subscale, there were significant differences in the stress score between parents (2.87) and nurses (3.35) $(\mathrm{t}=3.29, p=.001)$. Parents showed a lower stress score than nurses' perceptions for items including "too quick explanation" $(\mathrm{t}=6.11, p<.001)$, "can't understand terminology" $(\mathrm{t}=6.38, p<.001)$, "confusing explanation" ( $\mathrm{t}=3.78$, $p<.001)$, "insufficient explanation" $(\mathrm{t}=2.21, p=.029)$, and "too many staff talk to me" $(\mathrm{t}=4.02, p<.001)$ (Table 5).

\section{DISCUSSION}

This study aimed to identify and compare the level of stress felt by parents of VLBWIs and parents' stress perceived by NICU nurses in order to help nurses to understand parents of VLBWIs and to establish standards for appropriate care.

The mean stress score among parents of VLBWIs was 3.31, which was lower than the score of 3.45 for nurses' perception of parental stress. In general, nurses perceived the parents of preterm infants to be in greater stress than their actual stress scores. This is consistent with the results of previous studies that nurses are well aware of the stressors affecting parents 
Table 4. Perceived Stress of Parents of VLBWIs by Subscale According to Nurses' Characteristics $(N=78)$

\begin{tabular}{|c|c|c|c|c|c|c|c|c|c|c|}
\hline \multirow{2}{*}{ Characteristics } & \multirow{2}{*}{ Categories } & \multirow[t]{2}{*}{$\mathrm{n}(\%)$} & \multicolumn{2}{|c|}{$\begin{array}{l}\text { Sights and } \\
\text { sounds }\end{array}$} & \multicolumn{2}{|c|}{$\begin{array}{c}\text { Infant's } \\
\text { appearance } \\
\text { and behaviors }\end{array}$} & \multicolumn{2}{|c|}{$\begin{array}{l}\text { Parental role } \\
\text { alterations }\end{array}$} & \multicolumn{2}{|c|}{$\begin{array}{c}\text { Communication } \\
\text { with health care } \\
\text { providers }\end{array}$} \\
\hline & & & $\mathrm{M} \pm \mathrm{SD}$ & $\begin{array}{l}\text { tor F } \\
(p)\end{array}$ & $\mathrm{M} \pm \mathrm{SD}$ & $\begin{array}{l}\text { tor F } \\
(p)\end{array}$ & $\mathrm{M} \pm \mathrm{SD}$ & $\begin{array}{l}\text { tor } \mathrm{F} \\
(p)\end{array}$ & $\mathrm{M} \pm \mathrm{SD}$ & $\begin{array}{l}\mathrm{t} \text { or F } \\
(p)\end{array}$ \\
\hline Total stress & & $83(100.0)$ & $3.44 \pm 0.66$ & & $3.60 \pm 0.79$ & & $3.29 \pm 0.98$ & & $3.35 \pm 0.79$ & \\
\hline Age (year) & $\begin{array}{l}<26 \\
\geq 26 \&<30 \\
\geq 30 \&<36 \\
\geq 36\end{array}$ & $\begin{array}{r}25(32.1) \\
29(37.2) \\
15(19.2) \\
9(11.5)\end{array}$ & $\begin{array}{l}3.28 \pm 0.64 \\
3.39 \pm 0.56 \\
3.68 \pm 0.67 \\
3.62 \pm 0.90\end{array}$ & $\begin{array}{l}1.46 \\
(.232)\end{array}$ & $\begin{array}{l}3.47 \pm 0.75 \\
3.54 \pm 0.83 \\
3.76 \pm 0.63 \\
3.92 \pm 0.97\end{array}$ & $\begin{array}{l}0.97 \\
(.412)\end{array}$ & $\begin{array}{l}3.12 \pm 1.03 \\
3.28 \pm 1.10 \\
3.53 \pm 0.64 \\
3.38 \pm 0.93\end{array}$ & $\begin{array}{l}0.55 \\
(.650)\end{array}$ & $\begin{array}{l}3.25 \pm 0.85 \\
3.35 \pm 0.71 \\
3.33 \pm 0.50 \\
3.69 \pm 1.18\end{array}$ & $\begin{array}{l}0.68 \\
(.569)\end{array}$ \\
\hline Marital status & $\begin{array}{l}\text { Single } \\
\text { Married }\end{array}$ & $\begin{array}{l}54(69.2) \\
24(30.8)\end{array}$ & $\begin{array}{l}3.31 \pm 0.60 \\
3.73 \pm 0.70\end{array}$ & $\begin{array}{l}2.66 \\
(.010)\end{array}$ & $\begin{array}{l}3.56 \pm 0.81 \\
3.70 \pm 0.74\end{array}$ & $\begin{array}{l}0.71 \\
(.482)\end{array}$ & $\begin{array}{l}3.24 \pm 1.05 \\
3.39 \pm 0.79\end{array}$ & $\begin{array}{l}0.62 \\
(.537)\end{array}$ & $\begin{array}{l}3.29 \pm 0.81 \\
3.49 \pm 0.72\end{array}$ & $\begin{array}{l}1.04 \\
(.304)\end{array}$ \\
\hline With children & $\begin{array}{l}\text { No } \\
\text { Yes }\end{array}$ & $\begin{array}{l}61(78.2) \\
17(21.8)\end{array}$ & $\begin{array}{l}3.30 \pm 0.62 \\
3.92 \pm 0.57\end{array}$ & $\begin{array}{c}3.66 \\
(<.001)\end{array}$ & $\begin{array}{l}3.50 \pm 0.81 \\
3.97 \pm 0.62\end{array}$ & $\begin{array}{l}2.19 \\
(.032)\end{array}$ & $\begin{array}{l}3.18 \pm 1.02 \\
3.67 \pm 0.67\end{array}$ & $\begin{array}{l}1.85 \\
(.068)\end{array}$ & $\begin{array}{l}3.27 \pm 0.79 \\
3.66 \pm 0.73\end{array}$ & $\begin{array}{l}1.86 \\
(.067)\end{array}$ \\
\hline Career (year) & $\begin{array}{l}<5^{\mathrm{a}} \\
\geq 5 \&<10^{\mathrm{b}} \\
\geq 10^{\mathrm{c}}\end{array}$ & $\begin{array}{l}43(55.2) \\
20(25.6) \\
15(19.2)\end{array}$ & $\begin{array}{l}3.29 \pm 0.64 \\
3.48 \pm 0.59 \\
3.81 \pm 0.69\end{array}$ & $\begin{array}{c}3.86 \\
(.026) \\
a, b<c^{*}\end{array}$ & $\begin{array}{l}3.49 \pm 0.82 \\
3.60 \pm 0.73 \\
3.93 \pm 0.72\end{array}$ & $\begin{array}{l}1.73 \\
(.185)\end{array}$ & $\begin{array}{l}3.23 \pm 1.08 \\
3.21 \pm 0.96 \\
3.57 \pm 0.64\end{array}$ & $\begin{array}{l}0.75 \\
(.474)\end{array}$ & $\begin{array}{l}3.28 \pm 0.89 \\
3.20 \pm 0.46 \\
3.78 \pm 0.71\end{array}$ & $\begin{array}{l}2.96 \\
(.058)\end{array}$ \\
\hline $\begin{array}{l}\text { NICU career } \\
\text { (year) }\end{array}$ & $\begin{array}{l}<5^{\mathrm{a}} \\
\geq 5 \&<10^{\mathrm{b}} \\
\geq 10^{\mathrm{c}}\end{array}$ & $\begin{array}{l}45(57.7) \\
19(24.3) \\
14(18.0)\end{array}$ & $\begin{array}{l}3.27 \pm 0.63 \\
3.53 \pm 0.57 \\
3.86 \pm 0.69\end{array}$ & $\begin{array}{c}4.91 \\
(.010) \\
a, b<c^{*}\end{array}$ & $\begin{array}{l}3.50 \pm 0.80 \\
3.60 \pm 0.75 \\
3.95 \pm 0.75\end{array}$ & $\begin{array}{l}1.79 \\
(.174)\end{array}$ & $\begin{array}{l}3.23 \pm 1.06 \\
3.18 \pm 0.97 \\
3.62 \pm 0.63\end{array}$ & $\begin{array}{l}1.01 \\
(.369)\end{array}$ & $\begin{array}{l}3.30 \pm 0.87 \\
3.17 \pm 0.45 \\
3.79 \pm 0.73\end{array}$ & $\begin{array}{l}2.98 \\
(.057)\end{array}$ \\
\hline $\begin{array}{l}\text { Working } \\
\text { position }\end{array}$ & $\begin{array}{l}\text { Staff nurse } \\
\geq \text { Charge nurse }\end{array}$ & $\begin{array}{l}63(80.8) \\
15(19.2)\end{array}$ & $\begin{array}{l}3.35 \pm 0.62 \\
3.81 \pm 0.69\end{array}$ & $\begin{array}{l}2.53 \\
(.013)\end{array}$ & $\begin{array}{l}3.53 \pm 0.79 \\
3.93 \pm 0.72\end{array}$ & $\begin{array}{l}1.80 \\
(.075)\end{array}$ & $\begin{array}{l}3.22 \pm 1.03 \\
3.57 \pm 0.64\end{array}$ & $\begin{array}{l}1.23 \\
(.222)\end{array}$ & $\begin{array}{l}3.25 \pm 0.77 \\
3.78 \pm 0.71\end{array}$ & $\begin{array}{l}2.42 \\
(.018)\end{array}$ \\
\hline $\begin{array}{l}\text { Education } \\
\text { level }\end{array}$ & $\begin{array}{l}\leq \text { Bachelor's degree graduate } \\
\geq \text { Master's degree graduate }\end{array}$ & $\begin{array}{l}71(91.0) \\
7(9.0)\end{array}$ & $\begin{array}{l}3.42 \pm 0.67 \\
3.60 \pm 0.59\end{array}$ & $\begin{array}{l}0.68 \\
(.499)\end{array}$ & $\begin{array}{l}3.56 \pm 0.78 \\
4.02 \pm 0.84\end{array}$ & $\begin{array}{l}1.45 \\
(.150)\end{array}$ & $\begin{array}{l}3.26 \pm 0.99 \\
3.56 \pm 0.82\end{array}$ & $\begin{array}{l}0.78 \\
(.449)\end{array}$ & $\begin{array}{l}3.31 \pm 0.78 \\
3.75 \pm 0.84\end{array}$ & $\begin{array}{l}1.42 \\
(.160)\end{array}$ \\
\hline
\end{tabular}

*Scheffé test, $p<.050$; NICU, neonatal intensive care unit; VLBWIs, very low birth weight infants.

of preterm infants [20]. The PSS:NICU score of parents of VLBWIs was lower than the stress score (3.6) among mothers of neonates in the NICU reported by Kim [19] who used the PSS tool. In the study conducted by Oh et al. [13], the PSS score of parents with severely ill neonates was lower than 3.5 points. This was slightly higher than the stress score of 3.23 points for the parents of VLBWIs in the NICU in Turkey [21]. The results of Baia et al. [15], who studied the parental stress of fathers and mothers of VLBWIs using the PSS, showed that mothers scored 4.0, while fathers scored 3.0, with lower PSS scores for fathers in all subscales. Fathers accounted for more than $39 \%$ of the parent subjects in this study, which might explain why the stress score was lower than in previous studies. We can speculate that parents of VLBWIs have less stress due to the decreased neonatal mortality and increased survival rate of VLBWIs owing to advances in medical technology and improved quality of care [8], but further research is needed to determine the specific factors contributing to their lower stress.

Mothers had significantly higher stress scores on the parental role alterations subscale, which is in line with previous studies $[13,15,22]$. The higher average stress score among mothers than fathers of preterm infants in the NICU is also con- sistent with the results of a study on stress among parents (mothers: 3.65 points, fathers: 3.07 points) of patients in the NICU by Lee et al. [4]. The same result was found in a study on stress among parents of NICU patients in Portugal (mothers: 4.0 points, fathers: 3.0 points) by Baia et al. [15]. Kim and Tak [23] reported that the maternal attachment score among mothers of preterm infants in the NICU (95.01) was markedly higher than that of parents of full-term babies, suggesting that maternal attachment plays a role in stress. Mothers are the primary caregivers, and since parenting stress felt by mothers can have a negative impact on the growth and development of infants, interventions are necessary for parents of VLBWIs, especially mothers.

However, the most important finding of this study relates to the infant's appearance and behaviors subscale. The parental stress perceived by nurses (3.60) was lower than parents' actual stress (3.78) in the infant's appearance and behaviors subscale, calling for further attention and research (Table 5). This is because VLBWIs receive NICU care as they are substantially smaller than full-term babies and have extremely immature body organs [4]. Most nurses know that VLBWIs' appearance changes as they grow, but parents may not be 
Table 5. Comparison of Stress of Parents with VLBWIs and NICU Nurses' Perceived Parental Stress $(N=161)$

\begin{tabular}{|c|c|c|c|c|}
\hline \multirow{2}{*}{ Variables } & \multirow{2}{*}{$\begin{array}{c}\text { Parents }(\mathrm{n}=83) \\
\mathrm{M} \pm \mathrm{SD}\end{array}$} & \multirow{2}{*}{$\begin{array}{c}\text { Nurse }(n=78) \\
M \pm S D\end{array}$} & \multirow{2}{*}{$\mathrm{t}$} & \multirow{2}{*}{$p$} \\
\hline & & & & \\
\hline Sights and sounds & $2.75 \pm 0.92$ & $3.44 \pm 0.66$ & 5.48 & $<.001$ \\
\hline Noise monitors / equipment & $2.67 \pm 1.32$ & $3.37 \pm 0.85$ & 4.01 & $<.001$ \\
\hline See monitors / equipment & $2.99 \pm 1.18$ & $3.79 \pm 0.93$ & 4.82 & $<.001$ \\
\hline Sudden noise / alarm & $3.60 \pm 1.17$ & $4.19 \pm 0.87$ & 3.65 & $<.001$ \\
\hline Other such babies & $2.92 \pm 1.08$ & $3.18 \pm 0.88$ & 1.69 & .093 \\
\hline Large number of staff & $1.58 \pm 1.40$ & $2.65 \pm 1.02$ & 5.61 & $<.001$ \\
\hline Infant's behavior and appearance & $3.78 \pm 0.71$ & $3.60 \pm 0.79$ & 1.45 & .149 \\
\hline Tubes / equipment & $3.13 \pm 1.16$ & $3.49 \pm 1.04$ & 2.04 & .043 \\
\hline Bruises /cuts /incisions & $3.95 \pm 0.95$ & $4.03 \pm 1.07$ & 0.46 & .643 \\
\hline Pale /jaundiced & $3.87 \pm 0.88$ & $3.31 \pm 1.05$ & 3.68 & $<.001$ \\
\hline Breathing pattern & $4.25 \pm 0.78$ & $3.88 \pm 0.81$ & 2.95 & .004 \\
\hline Unusual color & $4.25 \pm 0.79$ & $4.19 \pm 0.81$ & 0.48 & .631 \\
\hline Apnea & $4.72 \pm 0.57$ & $4.60 \pm 0.73$ & 1.16 & .246 \\
\hline Small size & $3.67 \pm 0.96$ & $3.59 \pm 1.00$ & 0.55 & .584 \\
\hline Wrinkled & $3.05 \pm 1.16$ & $2.88 \pm 1.03$ & 0.94 & .346 \\
\hline Ventilator & $3.71 \pm 1.01$ & $3.72 \pm 1.09$ & 0.04 & .966 \\
\hline Needles / tubes put in & $3.64 \pm 0.94$ & $3.69 \pm 1.24$ & 0.31 & .759 \\
\hline Intravenous / feed tube & $3.20 \pm 1.06$ & $3.01 \pm 1.22$ & 1.07 & .287 \\
\hline Pain & $4.12 \pm 0.99$ & $3.74 \pm 1.02$ & 2.37 & .019 \\
\hline Crying for a long time & $3.72 \pm 1.07$ & $3.97 \pm 0.87$ & 1.63 & .106 \\
\hline Scared & $3.90 \pm 1.01$ & $3.63 \pm 1.09$ & 1.66 & .098 \\
\hline Sad & $3.80 \pm 1.04$ & $3.37 \pm 1.12$ & 2.48 & .014 \\
\hline Limp and weak & $4.06 \pm 0.93$ & $3.58 \pm 1.08$ & 3.06 & .003 \\
\hline Jerky / restless & $4.23 \pm 0.82$ & $3.86 \pm 0.92$ & 2.70 & .008 \\
\hline Can't cry & $3.52 \pm 1.13$ & $3.18 \pm 1.02$ & 1.99 & .048 \\
\hline Percussion of his/her chest & $2.93 \pm 1.15$ & $2.76 \pm 1.06$ & 0.97 & .332 \\
\hline Parent's role alterations & $3.19 \pm 0.96$ & $3.29 \pm 0.98$ & 0.64 & .521 \\
\hline Separation & $3.63 \pm 1.17$ & $3.62 \pm 1.31$ & 0.06 & .955 \\
\hline Can't breastfeed & $3.46 \pm 1.17$ & $3.35 \pm 1.31$ & 0.57 & .569 \\
\hline Can't provide care & $3.23 \pm 1.23$ & $2.97 \pm 1.22$ & 1.33 & .186 \\
\hline Can't hold & $3.49 \pm 1.27$ & $3.47 \pm 1.26$ & 0.10 & .922 \\
\hline Can't imagine clearly & $2.95 \pm 1.32$ & $3.13 \pm 1.25$ & 0.87 & .385 \\
\hline Alone & $3.48 \pm 1.17$ & $3.31 \pm 1.18$ & 0.94 & .348 \\
\hline Helpless / can't protect & $3.94 \pm 0.99$ & $3.78 \pm 1.06$ & 0.97 & .332 \\
\hline Anxious to touch or hold & $2.94 \pm 1.27$ & $3.18 \pm 0.98$ & 1.35 & .180 \\
\hline Medical staff closer to the infant than me & $1.52 \pm 1.40$ & $2.68 \pm 1.11$ & 5.85 & $<.001$ \\
\hline Helpless / how to help & $3.27 \pm 1.30$ & $3.27 \pm 1.30$ & 0.70 & .483 \\
\hline Communication with health care providers & $2.87 \pm 1.06$ & $3.35 \pm 0.79$ & 3.29 & .001 \\
\hline Too quick explanation & $1.98 \pm 1.26$ & $3.04 \pm 0.93$ & 6.11 & $<.001$ \\
\hline Can't understand terminology & $2.14 \pm 1.24$ & $3.21 \pm 0.84$ & 6.38 & $<.001$ \\
\hline Confusing explanation & $3.08 \pm 1.33$ & $3.77 \pm 0.95$ & 3.78 & $<.001$ \\
\hline Insufficient explanation & $3.28 \pm 1.29$ & $3.65 \pm 0.83$ & 2.21 & .029 \\
\hline Incomplete description & $3.34 \pm 1.36$ & $3.68 \pm 0.88$ & 1.91 & .058 \\
\hline Too many staff talk to me & $2.01 \pm 1.37$ & $2.78 \pm 1.05$ & 4.02 & $<.001$ \\
\hline Can't help me when I visit or call & $3.17 \pm 1.33$ & $3.33 \pm 1.02$ & 0.89 & .378 \\
\hline Can't trust staff & $3.35 \pm 1.49$ & $3.46 \pm 1.07$ & 0.55 & .581 \\
\hline Seem worried about my infant & $3.06 \pm 1.53$ & $3.19 \pm 1.31$ & 0.59 & .559 \\
\hline They don't want me to be there & $2.94 \pm 1.29$ & $3.27 \pm 1.08$ & 1.75 & .082 \\
\hline They don't understand my infant's behavior or special needs & $3.25 \pm 1.36$ & $3.51 \pm 1.03$ & 1.37 & .172 \\
\hline
\end{tabular}

NICU, neonatal intensive care unit; VLBWIs, very low birth weight infants. 
aware of this.

Kim [19] found that the highest perceived stress level was 3.9 for the infant's appearance and behaviors subscale, whereas a score of only 3.5 was found for the parental role alterations subscale. In our study, in the infant's appearance and behaviors subscale, the highest score (4.72) was found for the "infant apnea" item. Parents who witness their infant stop breathing become flustered and have questions about the survival of the infant; nurses should understand this concern and provide a more detailed explanation about the nursing procedures and the infant's status.

Lee et al. [4] reported that parents showed significantly higher stress scores than nurses' perceived scores for the "pale /jaundiced," "breathing pattern," "pain," "sad," "limp and weak," "jerky / restless," and "can't cry" items, suggesting that parents should express their emotions regarding these factors and nurses should support them and provide detailed explanations. However, parents showed the greatest stress for "mechanical ventilation" (4.0) in the study by Kim [19] among parents of NICU-admitted infants, "the infant looks sick" in the study by $\mathrm{Oh}$ et al. [13] among parents of severely ill infants, and "the infant suddenly shivers or moves unstably" in a study among parents of preterm infants. In the study by Lee et al. [4], parents were most stressed about their relationship with the infant and changes in the parental role, which reflects a slight difference from our findings.

In contrast to the results of previous studies finding that parents showed the greatest stress in the parental role alterations subscale $[5,18]$, our results showed that stress in the parental role alterations subscale was the second-highest. But these results nevertheless suggest that parents of VLBWIs are concerned and stressed about parental role alterations. Thus, parents should be given education and explanations about parental role alterations so that they can prepare accordingly.

Alkozei et al. [16] reported that nurses perceived that an unfamiliar environment, continuous alarm sounds, and other noise, as well as ineffective communication with health care providers, would cause parents to be highly stressed. However, parents were more stressed about the infant's appearance, behaviors, and treatment, as well as challenges in parental role alterations, than environmental factors. The study of Mizrak et al. [24] found that because parents were only allowed to meet their infant for short durations and frequencies, they were further stressed about the infant's status at the time of their visits. Moreover, the parents of VLBWIs experience elevated stress levels because these infants are lighter than normal and preterm, have immature organs, and undergo various invasive procedures along with mechanical ventilation.

Furthermore, the parents of NICU-admitted VLBWIs experienced spikes in their stress level due to their anxiety about not being able to be with the infant instead of being prepared to adjust to changes in their parental role. In light of the results of previous studies that parents' stress increases with an increasing length of stay of the infant [25] and that physical separation from their infant can intensify parents' emotional vulnerability [16], nurses should educate parents regarding parental role alterations such that their future parenting behaviors are not affected. According to an analysis of sources of stress for the parents of preterm newborns in the study of Kumar and Mallick [9], early involvement of mothers in neonatal care, feeding the infant, skin-to-skin contact, and allowing visits by close family members may play a pivotal role in reducing mothers' stress levels.

This study was conducted among parents of VLBWIs admitted to the NICU in three university- affiliated hospitals and nurses in these NICUs, so the findings have limited generalizability. The comparison of the two groups was limited because extremely low birth weight infants are rare. The average age and career experience of nurses were relatively high because which requires sufficient career experience to take care of VLBWIs. Therefore, the nurses of this study are representative of nurses caring for VLBWIs, but not all NICU nurses.

\section{CONCLUSION}

This study is significant in that it provides a standard for how nurses should educate parents to reduce parental stress. Because parents' stress related to the appearance and behavior of the infant was significantly higher than the nurse's perceptions, nurses should understand the stress of parents of VLBWIs to support them and help them fulfill their parental role alterations. Investigating the difference between nurses' perceptions of parents' stress and actual parental stress is important for identifying the greatest stressors. Nursing support is needed regarding the infant's appearance and behaviors subscale and parental role alterations subscale. NICU nurses should understand the stressors and the severity of stress suffered by parents and provide care to relieve that stress. It is important for nurses to provide additional explanations about the appearances of VLBWIs and nursing procedures, as well as the NICU environment, and provide tailored education that prepares parents for their parental roles.

\section{ORCID}

Seol-Hee Moon

Ho-Ran Park

Dong Yeon Kim

https://orcid.org/0000-0002-4188-3677

https://orcid.org/0000-0003-0867-1979

https://orcid.org/0000-0001-9500-5792 


\section{Authors' contribution}

Conceptualization: Seol-Hee Moon, Ho-Ran Park; Data collection, Formal analysis: all authors; Writing-original draft: Seol-Hee Moon; Writing-review and editing: all authors; Final approval of published version: all authors.

\section{Conflict of interest}

No existing or potential conflict of interest relevant to this article was reported.

\section{Funding}

None.

\section{Data availability}

Please contact the corresponding author for data availability.

\section{Acknowledgements}

None.

\section{REFERENCES}

1. Choi MH, Kang IS, Kim YH. Effects of hearing recorded mother's voice on physiological reactions and behavioral state of sleep, weight of very low birth weight infants. Child Health Nursing Research. 2014;20(3):185-195.

https://doi.org/10.4094/chnr.2014.20.3.185

2. Joo SH, Kim TI. Noise level and frequency experienced by premature infants receiving incubator care in the neonatal intensive care unit. Child Health Nursing Research. 2020;26(2):296-308. https://doi.org/10.4094/chnr.2020.26.2.296

3. Lee HS, Kang SJ. Factors influencing post-traumatic growth in mothers with premature infants admitted to the neonatal intensive care unit. Child Health Nursing Research. 2020;26(2):267-276. https://doi.org/10.4094/chnr.2020.26.2.267

4. Lee $\mathrm{CH}$, Jang MH, Choi YS, Shin H. The relationship between parental stress and nurses' communication as perceived by parents of high-risk newborns. Child Health Nursing Research. 2019;25(2): 184-195. https://doi.org/10.4094/chnr.2019.25.2.184

5. Han SY, Chae SM. Perceived parental stress and nursing support for fathers of high risk infants. Child Health Nursing Research. 2016;22(3):190-198. https://doi.org/10.4094/chnr.2016.22.3.190

6. Bry A, Wigert H. Psychosocial support for parents of extremely preterm infants in neonatal intensive care: A qualitative interview study. Bry and Wigert BMC Psychology. 2019;7(1):76.

https://doi.org/10.1186/s40359-019-0354-4
7. Prouhet PM, Gregory MR, Russell CL, Yaeger LH. Fathers' stress in the neonatal intensive care unit: A systematic review. Advances in Neonatal Care. 2018;18(2):105-120.

https://doi.org/10.1097/ANC.0000000000000472

8. Lee SJ, Choi EK, Park J, Kim HS. Correlations between a flexible parental visiting environment and parental stress in neonatal intensive care units. Child Health Nursing Research. 2019;25(4): 377-387. https://doi.org/10.4094/chnr.2019.25.4.377

9. Kumar N, Mallick AK. Maternal stress in neonatal intensive care unit very often overlooked by health professionals. Industrial Psychiatry Journal. 2020;29(1):130-133. https://doi.org/10.4103/ipj.ipj_88_19

10. Choi E, Lee Y. A mother's experience of hospitalization of her newborn in the neonatal intensive care unit. Child Health Nursing Research. 2018;24(4):407-419. https://doi.org/10.4094/chnr.2018.24.4.407

11. Yu M. Work stress, turnover intention and burnout among nurses in neonatal intensive care unit. Journal of Korean Academy of Nursing Administration. 2011;17(1):115-126. https://doi.org/10.11111/jkana.2011.17.1.115

12. Im M, Oh J. Nursing support perceived by mothers of preterm infants in a neonatal intensive care unit in South Korea. Child Health Nursing Research. 2021;27(2):146-159. https://doi.org/10.4094/chnr.2021.27.2.146

13. Oh KS, Lim JY, Cho IS, Ham OK. A comparison of parent stress sick newborn in NICU. Journal of Korean Academic Society of Home Health Care Nursing. 2010;17(1):12-20.

14. Tandberg BS, Sandtrø HP, Vårdal M, Rønnestad A. Parents of preterm evaluation of stress and nursing support. Journal of Neonatal Nursing. 2013;19(6):317-326. https://doi.org/10.1016/j.jnn.2013.01.008

15. Baia I, Amorim M, Silva S, Kelly-Irving M, de Freitas C, Alves E. Parenting very preterm infants and stress in Neonatal Intensive Care Units. Early Human Development. 2016;101:3-9. https://doi.org/10.1016/j.earlhumdev.2016.04.001

16. Alkozei A, McMahon E, Lahav A. Stress levels and depressive symptoms in NICU mothers in the early postpartum period. Journal of Maternal-Fetal and Neonatal Medicine. 2014;27(17):17381743. https://doi.org/10.3109/14767058.2014.942626

17. Faul F, Erdfelder E, Buchner A, Lang AG. Statistical power analyses using $\mathrm{G}^{*}$ Power 3.1: Tests for correlation and regression analyses. Behavior Research Methods. 2009;41(4):1149-1160. https://doi.org/10.3758/BRM.41.4.1149

18. Miles MS, Funk SG, Carlson J. Parental stressor scale: Neonatal intensive care unit. Nursing Research. 1993;42(3):148-152. https://doi.org/10.1097/00006199-199305000-00005

19. Kim TI. A study on the perceived stress level of mothers in the neonatal intensive care unit patients. Child Health Nursing Research. 2000;6(2):224-239.

20. Polit DF, Beck CT, Owen SV. Is the CVI an acceptable indicator of 
content validity? Appraisal and recommendations. Research in Nursing ana Health. 2007;30(4):459-467.

https://doi.org/10.1002/nur.20199

21. Pritchard VE, Montgomery-Honger A. A comparison of parent and staff perceptions of setting-specific and everyday stressors encountered by parents with very preterm infants experiencing neonatal intensive care. Early Human Development. 2014;90(10):549-555.

https://doi.org/10.1016/j.earlhumdev.2014.07.006

22. Heydarpour S, Keshavarz Z, Bakhtiari M. Factors affecting adaptation to the role of motherhood in mothers of preterm infants admitted to the neonatal intensive care unit: A qualitative study. Journal of Advanced Nursing. 2017;73(1):138-148. https://doi.org/10.1111/jan.13099
23. Kim AR, Tak YR. Maternal role development in neonatal intensive care unit graduate mothers of premature infant. Korean Journal of Women Health Nursing. 2015;21(4):308-320.

https://doi.org/10.4069/kjwhn.2015.21.4.308

24. Mizrak B, Deniz AO, Acikgoz A. Anxiety levels of mothers with newborns in a neonatal intensive care unit in Turkey. Pakistan Journal of Medical Sciences. 2015;31(5):1176-1181. https://doi.org/10.12669/pjms.315.7792

25. Pichler-Stachl E, Pichler G, Baik N, Urlesberger B, Alexander A, Urlesberger $\mathrm{P}$, et al. Maternal stress after preterm birth: Impact of length of antepartum hospital stay. Women and Birth. 2016;29(6): e105-e109. https://doi.org/10.1016/j.wombi.2016.04.008 